\title{
Flowering plants of Sarkuwa VDC, Baglung, Western Nepal
}

\author{
Indramani Bhagat* and Bimala Subedi \\ Department of Botany, Post Graduate Campus, T.U., Biratnagar, Nepal \\ "E-mail: drimbhagat@yahoo.com
}

\begin{abstract}
The study was conducted in Sarkuwa VDC of Baglung district, Western Nepal to explore and document the flowering plants. Altogether 127 species of plants belonging to108 genera and 53 families were collected. Dominant families of the study area are Moraceae with 11 species, Asteraceae with 9 species, Fabaceae and Euphorbiaceae with 8 species, Lamiaceae and Verbenaceae with 5 species and Anacardiceae, Meliaceae, Poaceae with 4 species. Among the 127 species, trees are dominant in species composition attaining 39.37 of the whole floristic value, shrub with $27.55 \%$, herb with $25.19 \%$, and climber with $5.15 \%$, under shrub with $1.57 \%$ and finally parasite by attaining $0.78 \%$.
\end{abstract}

Key words: Dominant families, Floristic value, Medicinal plants

\section{Introduction}

Nepal is a small country occupying an area of 147,181 sq $\mathrm{km}$ and lying between Lat. $26^{\circ} 22^{\prime} \mathrm{N}$ to $30^{\circ} 27^{\prime} \mathrm{N}$ and Long. $80^{\circ} 4^{\prime} \mathrm{E}$ to $88^{\circ} 12^{\prime} \mathrm{E}$, is well known as natures paradise for its rich biodiversity. Due to the great geographic along with climatic variation, Nepal is endowed with the tropical to alpine vegetation (Chaudhary, 1998). Nepal offers unique opportunity to conserve biodiversity of many phytogeographical provinces in this small territory. It has biological richness of both the Indo-malaya and Paler tic realm including endemic Himalayan flora.

Nepal's position in the central sector of Himalaya is that of a transitional zone of interpretation between two differing environment of the Eastern Himalaya and Western Himalaya (Shrestha \& Joshi, 1996). Thus Nepal offers unique opportunity to conserve biodiversity of many phytogeographical provinces in this small territory. It has biological richness of both the Indo-malaya and Palerctic realm including endemic Himalayan flora. In Nepal, scientist have identified 118 ecosystems, 75 types of vegetation and 35 types of forest harboring more than 6500 species of flowering plants (Stainton, 1972). Of the total estimated 6500 species of flowering plants, about $4 \%$ are endemic to the country and $30 \%$ are endemic to the Himalayas. Although Nepal shares about $0.09 \%$ of all world total land by area, its share in world's total flowering plants species is more than $2 \%$. Nepal occupies the 10th position on richness of flowering plants diversity in Asia, the number of flowering plants enumerated in Nepal is 6067 belonging to 216 families and 1534 genera (Press et al., 2000).

There are a few reports describing flowering plants from Bukini VDC of Baglung discrict (Sapkota, 2000) but not from Sarkuwa VDC. So, present work is based to provide introductory knowledge of flowering plants of Sarkuwa VDC of Baglung district, Western Nepal. 


\section{Materials and Methods}

The flowering plants of Sarkuwa VDC of Baglung district were collected. The herbarium was prepared and specimens identified swith the help of available literatures. (Haines, 1961; Lawrence, 1965; Hara et al., 1978, 1979, 1982; Hooker, 1883-1897, 1854; Siwakoti \& Varma, 1996, 1999) as well as from herbarium sector. The identified species were deposited at Post Graduate Campus, Biratnagar.

\section{Results and Discussion}

From the study area altogether 127 species belonging to 108 genera and 53 families were reported. Trees are the dominant attaining $39.37 \%$ of the whole floristic composition followed by shrub with $27.55 \%$, herb with $25.19 \%$, climber with $5.15 \%$, under shrub with $1.57 \%$ and parasite by attaining $0.78 \%$ (Table 1 ).

Table 1. Number and percentage of plant species on the basis of their life form.

\begin{tabular}{clcc}
\hline S.N. & Life form (Habit) & Total number & Percentage ( \%) \\
\hline 1 & Tree & 50 & 39.37 \\
2 & Shrub & 35 & 27.55 \\
3 & Herb & 32 & 25.19 \\
4 & Climber & 7 & 5.15 \\
5 & Under shrub & 2 & 1.57 \\
6 & Parasite & 1 & 0.78 \\
\hline
\end{tabular}

The dominant families of the study area are Moraceae with 11 species, Asteraceae with 9 species, Fabaceae and Euphorbiaceae with 8 species, Lamiaceae and Verbenaceae with 5 species, Anacardiceae, Meliaceae and Poaceae with 6 species (Table 2).

Table 2. Family, genera and species with their habit, local name, flowering and fruiting period, altitude and uses.

\begin{tabular}{|c|c|c|c|c|c|}
\hline Botanical name / Family & Habit & $\begin{array}{l}\text { Local name } \\
\text { (Nepali) }\end{array}$ & $\begin{array}{l}\text { Flower- } \\
\text { fruit } \\
\text { period }\end{array}$ & $\begin{array}{l}\text { Altitude } \\
\quad(\mathbf{m})\end{array}$ & Parts/Used in \\
\hline $\begin{array}{l}\text { Justicia dhatoda Nees./ } \\
\text { Acanthaceae }\end{array}$ & $S$ & Asuro & Jan-Jul & 1200 & $\begin{array}{l}\text { Plant \& leaves juice/ Heart } \\
\text { trouble, fever, jaundice, kill } \\
\text { worms in intestine, cuts, cough }\end{array}$ \\
\hline $\begin{array}{l}\text { Strobilanthes atropurpureus } \\
\text { Nees }\end{array}$ & & Aankhle & Jan- Mar & 1600 & $\begin{array}{l}\text { Leaf/ Food for Lepidoptera } \\
\text { larvae }\end{array}$ \\
\hline $\begin{array}{l}\text { Alternanthera sessilis L./ } \\
\text { Amaranthaceae }\end{array}$ & $\mathrm{H}$ & Bhiringi jhar & $\begin{array}{l}\text { All } \\
\text { months }\end{array}$ & 1200 & $\begin{array}{l}\text { whole plant/Diarrhoea, } \\
\text { dysentery }\end{array}$ \\
\hline Amaranthus spinosus L. & $\mathrm{H}$ & Kade lude & Jul-Nov & 1200 & $\begin{array}{l}\text { whole plant/ Stomach } \\
\text { gonorrhea eczema }\end{array}$ \\
\hline Amaranthus viridis L. & $\mathrm{H}$ & Lude sag & Jan-Aug & 1200 & $\begin{array}{l}\text { Seeds/ Diarrhoea, blood } \\
\text { purifier, mouth sore }\end{array}$ \\
\hline $\begin{array}{l}\text { Choerospondias axillaris } \\
\text { Roxb./ Anacardiaceae }\end{array}$ & $\mathrm{T}$ & Lapsi & Feb-Aug & 1200 & $\begin{array}{l}\text { Fruit \& seeds/ Pickles, spicy } \\
\text { candy, fuel }\end{array}$ \\
\hline Rhus javanica Miller. & $\mathrm{T}$ & Bhaki amilo & Feb-Sep & 1220 & $\begin{array}{l}\text { Fruit/ Coughs, diarrhoea, } \\
\text { dysentery, uterine bleedings }\end{array}$ \\
\hline $\begin{array}{l}\text { Semecarpus anacardium } \\
\text { Linn. }\end{array}$ & $\mathrm{T}$ & Bhalayo & May-oct & 1250 & $\begin{array}{l}\text { Fruits \& nut extract/ Epilepsy, } \\
\text { rheumatism, , skin disorders, } \\
\text { fever, worm infestation, hair }\end{array}$ \\
\hline
\end{tabular}




\begin{tabular}{|c|c|c|c|c|c|}
\hline Spondias pinnata (Lf) Kurz & $\mathrm{T}$ & Ambra & Apr-Sep & 850 & $\begin{array}{l}\text { Fruit, leaves, flowers \& bark/ } \\
\text { Pickles, jams, stomach aches, } \\
\text { dysentery, rheumatism }\end{array}$ \\
\hline $\begin{array}{l}\text { Agave americana L./ } \\
\text { Agavaceae }\end{array}$ & $\mathrm{S}$ & Kettuke & $\begin{array}{l}\text { Once in } \\
\text { years }\end{array}$ & 1050 & $\begin{array}{l}\text { Whole plant/ Wound healing, } \\
\text { burns, ulcers, tuberculosis }\end{array}$ \\
\hline $\begin{array}{l}\text { Plumeria rubra L./ } \\
\text { Apocynaceae }\end{array}$ & $\mathrm{T}$ & $\begin{array}{l}\text { Golaicha } \\
\text { (Red jasmine) }\end{array}$ & $\begin{array}{l}\text { Whole } \\
\text { year }\end{array}$ & 650 & $\begin{array}{l}\text { Leaves, flower, bark/ Itch, } \\
\text { rheumatism, gum troubles, } \\
\text { fever, dysentery }\end{array}$ \\
\hline $\begin{array}{l}\text { Braciopsis hainla (Buch.- } \\
\text { Ham.ex D. Don)/ Araliaceae }\end{array}$ & S & Chuletro & Dec-Aug & 1300 & Whole plant/ Fodder \\
\hline $\begin{array}{l}\text { Calotropis gigantia L./ } \\
\text { Asclepiadaceae }\end{array}$ & $\mathrm{S}$ & Aank & $\begin{array}{l}\text { Whole } \\
\text { year }\end{array}$ & 700 & $\begin{array}{l}\text { Latex, root bark, flowers/ } \\
\text { Dysentery, cold, cough, asthma }\end{array}$ \\
\hline $\begin{array}{l}\text { Ageratum conizoids L./ } \\
\text { Asteraceae }\end{array}$ & $\mathrm{H}$ & Ilame Jhar & Dec-May & 600 & $\begin{array}{l}\text { Whole plant/ Bleeding, } \\
\text { rheumatism, weed }\end{array}$ \\
\hline Artemisia dubia Wall. & US & Titepati & Aug-Oct & 1350 & $\begin{array}{l}\text { Leaves, roots/ Respiratory tract } \\
\text { infections, asthma, gastric }\end{array}$ \\
\hline $\begin{array}{l}\text { Bedens pilosa var. bipinnata } \\
\text { (L.) Hook. f }\end{array}$ & & Kuro & May-Oct & 700 & $\begin{array}{l}\text { Young shoot \& leaves/ Kidney } \\
\text { problems, food }\end{array}$ \\
\hline $\begin{array}{l}\text { Blumea } \\
\text { hieraciifolia(D.Don)DC }\end{array}$ & $\mathrm{H}$ & Sahasrabuti & Sept.-Mar & 1250 & $\begin{array}{l}\text { Whole plant/ Treatment of } \\
\text { infection }\end{array}$ \\
\hline $\begin{array}{l}\text { Ageratina adenophora (L.) } \\
\text { King \& Robinson }\end{array}$ & $\mathrm{H}$ & Banmara & Sept.-Ma1 & 1300 & Leaf/ Scabies \\
\hline $\begin{array}{l}\text { Gnaphalium polycaulon } \\
\text { Pers (Cudweeds) }\end{array}$ & $\mathrm{H}$ & Bukijhar & Jan-Apr. & 1300 & $\begin{array}{l}\text { Whole plant/ Food for } \\
\text { caterpillars }\end{array}$ \\
\hline $\begin{array}{l}\text { Inula cappa (Buch-Hamex } \\
\text { D.Don) DC }\end{array}$ & $\mathrm{S}$ & Gai tihara & May-Oct & 700 & $\begin{array}{l}\text { Leaf/ Respitory, digestive, } \\
\text { cancer, microbial diseases }\end{array}$ \\
\hline Sonchus asper L. & $\mathrm{H}$ & Prickly & May-Oct & 1300 & Leaf, stem/ Raw, cooked \\
\hline Xanthium strumarium L. & $\mathrm{H}$ & Bhede kuro & Jan-Apr & 1200 & $\begin{array}{l}\text { Root/ Cooling, digestive, tonic } \\
\text { treat swollen bones \& fractures }\end{array}$ \\
\hline $\begin{array}{l}\text { Berberis aristata } \mathrm{DC} / \\
\text { Berberidaceae }\end{array}$ & $\mathrm{S}$ & Chutro & Apr-may & 1300 & $\begin{array}{l}\text { Bark, berries/Jaundice. piles, } \\
\text { eye diseases, gums, dysentery, } \\
\text { urinary infection }\end{array}$ \\
\hline $\begin{array}{l}\text { Alnus nepalensis D.Don/ } \\
\text { Betulaceae }\end{array}$ & $\mathrm{T}$ & Utis & May-Sep & 1550 & $\begin{array}{l}\text { Whole plant/ Reclamation, fire } \\
\text { wood, making charcoal }\end{array}$ \\
\hline $\begin{array}{l}\text { Bombax ceiba L./ } \\
\text { Bombacaceae }\end{array}$ & $\mathrm{S}$ & Simal & $\begin{array}{l}\text { Once in } \\
\text { year }\end{array}$ & 950 & $\begin{array}{l}\text { Whole plant/ Headache, cough, } \\
\text { cold, dysentery, influenza, } \\
\text { urinary infections }\end{array}$ \\
\hline $\begin{array}{l}\text { Chenopodium album L./ } \\
\text { Chenopodiaceae }\end{array}$ & $\mathrm{H}$ & Bethe & Nov-Feb & 700 & Leaf, fruit, root/ Anemia, fever \\
\hline $\begin{array}{l}\text { Terminalia alata Heyne ex } \\
\text { Roth/ Combretaceae }\end{array}$ & $\mathrm{T}$ & Saaj & Feb-Oct & 700 & $\begin{array}{l}\text { Whole plant/ Timber for carts, } \\
\text { boat building }\end{array}$ \\
\hline $\begin{array}{l}\text { Terminalia bellirica } \\
\text { (Gaertn.) Roxb. }\end{array}$ & $\mathrm{T}$ & Barro & May-Oct & 1350 & $\begin{array}{l}\text { Fruit/ Bronchitis, fever, } \\
\text { diarrhoea }\end{array}$ \\
\hline Terminalia chebula Retz. & $\mathrm{T}$ & Harro & Feb-Aug & 1100 & $\begin{array}{l}\text { Fruit/ Diarrhoea, asthma, } \\
\text { urinary disorder, fever }\end{array}$ \\
\hline $\begin{array}{l}\text { Cordia dichotoma J.R. } \\
\text { Forst./ Cordiaceae }\end{array}$ & $\mathrm{T}$ & Bohori & Feb-Aug & 1350 & $\begin{array}{l}\text { Bark, leaf/ Treatment of ulcer, } \\
\text { colic pain, fodder }\end{array}$ \\
\hline Ehretia laevis Roxb. & $\mathrm{S}$ & Datingel & Feb-Aug & 1150 & $\begin{array}{l}\text { Leaf, bark/ Muscular pains, } \\
\text { eczema, throat infection }\end{array}$ \\
\hline
\end{tabular}




\begin{tabular}{|c|c|c|c|c|}
\hline $\begin{array}{l}\text { Shorea robusta Gaertn/ } \\
\text { Dipterocarpaceae }\end{array}$ & $\mathrm{T}$ & Sakhuwa & Mar-Jul 850 & $\begin{array}{l}\text { Fruit, resin/ Diarrhoea, } \\
\text { antiseptic for skin diseases }\end{array}$ \\
\hline $\begin{array}{l}\text { Dioscorea bulbifera } \mathrm{L} . / \\
\text { Dioscoreaceae }\end{array}$ & $\mathrm{H}$ & Githe Tarul & Jul-Oct & $\begin{array}{l}\text { Tuber/ Diabetes, eczema, } \\
\text { Intestinal parasites }\end{array}$ \\
\hline $\begin{array}{l}\text { Diospyros malabarica } \\
\text { (Desx.)/ Ebenaceae }\end{array}$ & $\mathrm{T}$ & Teeju & Feb-Jun 1350 & Leaf, fruit/ Dye cloth black \\
\hline $\begin{array}{l}\text { Lyonia formosa (Wall.) } \\
\text { Hans-Mazz./ Ericaceae }\end{array}$ & $\mathrm{T}$ & Angeri & May-Oct 1250 & $\begin{array}{l}\text { Whole plant/ Food for } \\
\text { Lepidoptera larvae, ornamental }\end{array}$ \\
\hline $\begin{array}{l}\text { Rhododendron arboreum } \\
\text { var. album Wall. }\end{array}$ & $\mathrm{T}$ & Lali guras & May-Aug 1200 & $\begin{array}{l}\text { Flower, bark, leaf / Indigestion, } \\
\text { liver \& lung troubles }\end{array}$ \\
\hline $\begin{array}{l}\text { Bridelia retusa L./ } \\
\text { Euphorbiaceae }\end{array}$ & $\mathrm{T}$ & $\begin{array}{l}\text { Gayo (Sun } \\
\text { pati) }\end{array}$ & Apr-Oct 1200 & $\begin{array}{l}\text { Whole plant, leaf / Cuts \& } \\
\text { wounds, wood }\end{array}$ \\
\hline Euphorbia hirta L. & $\mathrm{H}$ & Dudhe jhar & $\begin{array}{l}\text { All } \\
\text { months }\end{array}$ & $\begin{array}{l}\text { Plant extract/ Diarrhoea, fever, } \\
\text { snake bite }\end{array}$ \\
\hline Jatropha curcas L. & $\mathrm{S}$ & Sajiwan & Jul-Dec & $\begin{array}{l}\text { Twigs, seed/ Toothbrush, } \\
\text { burns, vegetable }\end{array}$ \\
\hline ga indica Wight & $\mathrm{T}$ & Malata & Jun-sep & $\begin{array}{l}\text { Branches, petioles, fruit/ To get } \\
\text { relief from venereal sores }\end{array}$ \\
\hline $\begin{array}{l}\text { Mallotus philippensis (Lam) } \\
\text { Mull. Arg }\end{array}$ & $\mathrm{T}$ & Sindhure & Sep-Mar 600 & $\begin{array}{l}\text { Root, bark/diarrhoea,dysentery, } \\
\text { Anthelmintic, indigestion }\end{array}$ \\
\hline $\begin{array}{l}\text { Phyllanthus amarus } \\
\text { Schumach \& Thonn. }\end{array}$ & $\mathrm{H}$ & Bhui amala & $\begin{array}{l}\text { Whole } \\
\text { year }\end{array}$ & $\begin{array}{l}\text { Fruit/ Diarrhoea, } d \text { y } \\
\text { stomach pain, jaun }\end{array}$ \\
\hline Phyllanthus emblica L. & $\mathrm{T}$ & Amala & Apr-Sept 1300 & $\begin{array}{l}\text { Fruit, bark/ Dy: } \\
\text { constipation, st }\end{array}$ \\
\hline $\begin{array}{l}\text { Sapium insigne (Royle) } \\
\text { Benth. Ex. Hook.f. }\end{array}$ & $\mathrm{T}$ & Khirro & Feb-Mar 1350 & $\begin{array}{l}\text { Leaf, fruit/ Skin disesse, } \\
\text { constipation, wounds }\end{array}$ \\
\hline $\begin{array}{l}\text { Bauhinia vahlii Wight \& } \\
\text { Arn./ Fabaceae }\end{array}$ & $\mathrm{C}$ & Bhorla & Apr-Feb 1150 & Seed/ Snake bite \\
\hline Bauhinia purpurea L. & $\mathrm{T}$ & Tanki & Sep-Mar 800 & $\begin{array}{l}\text { Flowers, root, stem, bark/ } \\
\text { Constipation, diarrhoea, ulcer }\end{array}$ \\
\hline Bauhinia malabarica Roxb. & & Koiralo & May-Oct 800 & $\begin{array}{l}\text { Root, bark/ Cholera, wound } \\
\text { healing, dysentery }\end{array}$ \\
\hline Cassia sophera L. & $\mathrm{S}$ & Thulo Tapre & $\begin{array}{ll}\text { Jan-May } 650 \\
\end{array}$ & Leaf/ Bronchitis, ringworm \\
\hline Crotalaria pallida Act. & $\mathrm{H}$ & Runche & Sep-Dec 100 & $\begin{array}{l}\text { Leaf/ Menstrual disorder, } \\
\text { diarrhoea }\end{array}$ \\
\hline Dalbergia sissoo Roxb. & $\mathrm{T}$ & Sisau & Mar-June 750 & $\begin{array}{l}\text { Leaf, whole plant/ Gonorrhoea, } \\
\text { leprosy, wood }\end{array}$ \\
\hline Erythrina stricta Roxb. & $\mathrm{T}$ & Phadelo & Mar-Sep 1500 & $\begin{array}{l}\text { Leaf, flower, bark/ make rope, } \\
\text { tanning dyeing, constipation }\end{array}$ \\
\hline Mimosa pudica L. & $\mathrm{H}$ & Lajawati & Mar-Dec 800 & $\begin{array}{l}\text { Root, leaf/ Urinary complaints, } \\
\text { glandular swelling }\end{array}$ \\
\hline $\begin{array}{l}\text { Castanopsis indica (Roxb) } \\
\text { Miq./ Fagaceae }\end{array}$ & $\mathrm{T}$ & Katus & Apr-Sept 1300 & $\begin{array}{l}\text { Branches, stems, nuts/ Timber, } \\
\text { edible }\end{array}$ \\
\hline $\begin{array}{l}\text { Castanopsis tribuloids (Sm.) } \\
\text { A.DC. }\end{array}$ & & Musure katus & Apr-Sept 1500 & $\begin{array}{l}\text { Branches, stems, seeds/ Bed } \\
\text { logs in mushroom cultivation, } \\
\text { fuel, edible }\end{array}$ \\
\hline Quercus lanata Sm. & $\mathrm{T}$ & Bhajo & May-Oct 800 & $\begin{array}{l}\text { Bark, seeds/ Fodder, diarrhoea, } \\
\text { dysentery, muscular pain }\end{array}$ \\
\hline Xylosma controversum Clos & & Raju & Jan-Jul & Fruit, leaf/ Eidible, narcotic \\
\hline
\end{tabular}




\begin{tabular}{|c|c|c|c|}
\hline G. Forst./ Flacourtiaceae & & & sedative, antispasmodic \\
\hline $\begin{array}{l}\text { Dichroa febrifuga Lour./ } \mathrm{S} \\
\text { Hydrangenaceae }\end{array}$ & Basak & May-Oct 1300 & $\begin{array}{l}\text { Root, leaf/ Malaria, stomach } \\
\text { cancer, cold cough, indigestion }\end{array}$ \\
\hline $\begin{array}{l}\text { Engelhardia spicata Les. ex T } \\
\text { Blume/ Juglandaceae }\end{array}$ & Mauwa & Nov-Apr 1340 & $\begin{array}{l}\text { Bark, fruit/ Edible, light timber, } \\
\text { tannins }\end{array}$ \\
\hline $\begin{array}{l}\text { Anisomeles indica Kuntze./ H } \\
\text { Lamiaceae }\end{array}$ & $\begin{array}{l}\text { Raato } \\
\text { chaarpate }\end{array}$ & Aug-Jan 1400 & $\begin{array}{l}\text { Whole plant/Eczema, snake } \\
\text { bites, rheumatism, cold. fever, } \\
\text { gas pain, uterine infection }\end{array}$ \\
\hline $\begin{array}{l}\text { Colebrookea oppositifolia S } \\
\text { Sm. }\end{array}$ & Dhusure & Nov-May 1300 & $\begin{array}{l}\text { Leaf, root/ Skin diseases, nose } \\
\text { bleeding }\end{array}$ \\
\hline Elsholtzia blanda Benth. & Bantulsi & Feb-Oct 1500 & $\begin{array}{l}\text { Seeds, leaf/ Condiment for } \\
\text { food, cooked, cold, fever }\end{array}$ \\
\hline Hyptis suaveolens ( L.) Poit. H & Toolo mirre & May-Aug 850 & $\begin{array}{l}\text { Seed, leaf, shoot/ Diarrhea, } \\
\text { fever, headache }\end{array}$ \\
\hline $\begin{array}{l}\text { Rabdosia coetsa (Buch.- } \\
\text { Ham. Ex D. Don) }\end{array}$ & Mire & Feb-Jul & $\begin{array}{l}\text { Whole plant/ Oil, diarrhea, } \\
\text { abdominal pain, dysentery }\end{array}$ \\
\hline $\begin{array}{l}\text { Litsea cubeba (Lour.)/ } \\
\text { Lauraceae }\end{array}$ & Siltimur & Feb-Aug 1300 & $\begin{array}{l}\text { Whole plant/ Essential oil, } \\
\text { cosmetic, infection, skin tonic, } \\
\text { lung cancer }\end{array}$ \\
\hline $\begin{array}{l}\text { Litsea monopetala (Roxb.) } \mathrm{T} \\
\text { Pers. } \\
\end{array}$ & Kutı & Jan-Dec 1300 & $\begin{array}{l}\text { d/ Treatment of } \\
\text { oil, wood }\end{array}$ \\
\hline $\begin{array}{l}\text { Dendrophthoe falcate (L.f) } \mathrm{P} \\
\text { Eitting/ Loranthaceae }\end{array}$ & Aair & May-Oct 1500 & $\begin{array}{l}\text { lant/ Wound healing, } \\
\text {, ulcers }\end{array}$ \\
\hline $\begin{array}{l}\text { Woodfordia fruticosa (L.) } \\
\text { Kurz/ Lythraceae }\end{array}$ & Dhairo & Feb-Jun 800 & Cough, ulcers, wounds \\
\hline $\begin{array}{l}\text { Sida rhombifolia } \text { L./ } \\
\text { Malvaceae }\end{array}$ & Sano & Mar-Dec 800 & tism \\
\hline Urena lobata $\mathrm{L}$. & Bhede k & Jan-Dec 800 & ots, seeds/ Rheumatism \\
\hline $\begin{array}{l}\text { Melastoma melabathricum S } \\
\text { L./ Melastomataceae }\end{array}$ & Angeri & Mar-Dec 1050 & $\begin{array}{l}\text { Bark, root/ Diarrhea, skin } \\
\text { disease, dysentery }\end{array}$ \\
\hline Osbeckia nepalensis Hook. S & Seto chulsi & Aug-Dec 1050 & $\begin{array}{l}\text { Root, leaf, flower/ Diabetes, } \\
\text { foot sores of cattle }\end{array}$ \\
\hline $\begin{array}{l}\text { Osbeckia stellata Buch.- } \\
\text { Ham. ex D. Donl }\end{array}$ & Rato chulsi & July-Dec 1300 & $\begin{array}{l}\text { plant/ Inflammatory } \\
\text { s }\end{array}$ \\
\hline $\begin{array}{l}\text { Cipadessa baccifera (Roth) S } \\
\text { Miq./ Meliaceae }\end{array}$ & Paireti & Apr-Feb 900 & $\begin{array}{l}\text { Root, bark/ Indigestion, } \\
\text { dysentery, skin itch, cough, } \\
\text { bleeding, swelling of gums }\end{array}$ \\
\hline Melia azedarach L. & Bakaino & Mar-Jun 850 & Bark/ Body pain, headache \\
\hline Toona ciliata M. Roem. & Tooni & Jan- Nov. 1050 & Bark/ Dysentery, heal wounds \\
\hline $\begin{array}{l}\text { Trichilia connaroides } \\
\text { (Wight \& Arn.) Bentv. }\end{array}$ & $\begin{array}{l}\text { Aankha } \\
\text { taruwa }\end{array}$ & Feb-Apr 1300 & $\begin{array}{l}\text { Leaf, fruit, stem/ Cholera, } \\
\text { antimicrobial, scabies, eczema } \\
\text { \& bdominal pain }\end{array}$ \\
\hline $\begin{array}{l}\text { Stephania glandulifera } \\
\text { Miers/ Menispermaceae }\end{array}$ & Batul Pate & Mar-Jun 1350 & $\begin{array}{l}\text { Tuber/ Treatment of epilepsy, } \\
\text { tuberculosis }\end{array}$ \\
\hline $\begin{array}{l}\text { Tinospora sinensis (Lour.) C } \\
\text { Merr. }\end{array}$ & Gurjo & Mar-Jun 650 & Leaf, stem/ Tonic, diabetes \\
\hline $\begin{array}{l}\text { Artocarpus lakoocha Wall. T } \\
\text { ex Roxb./ Moraceae }\end{array}$ & Badahar & Mar-Jun 1300 & $\begin{array}{l}\text { Wood, bark, leaf/ Treatment of } \\
\text { tapeworm, stomach ailments }\end{array}$ \\
\hline Ficus auriculata Lour. & Timilo & Apr-Aug 1300 & Leaf/ Diarrhea, dysentery \\
\hline
\end{tabular}




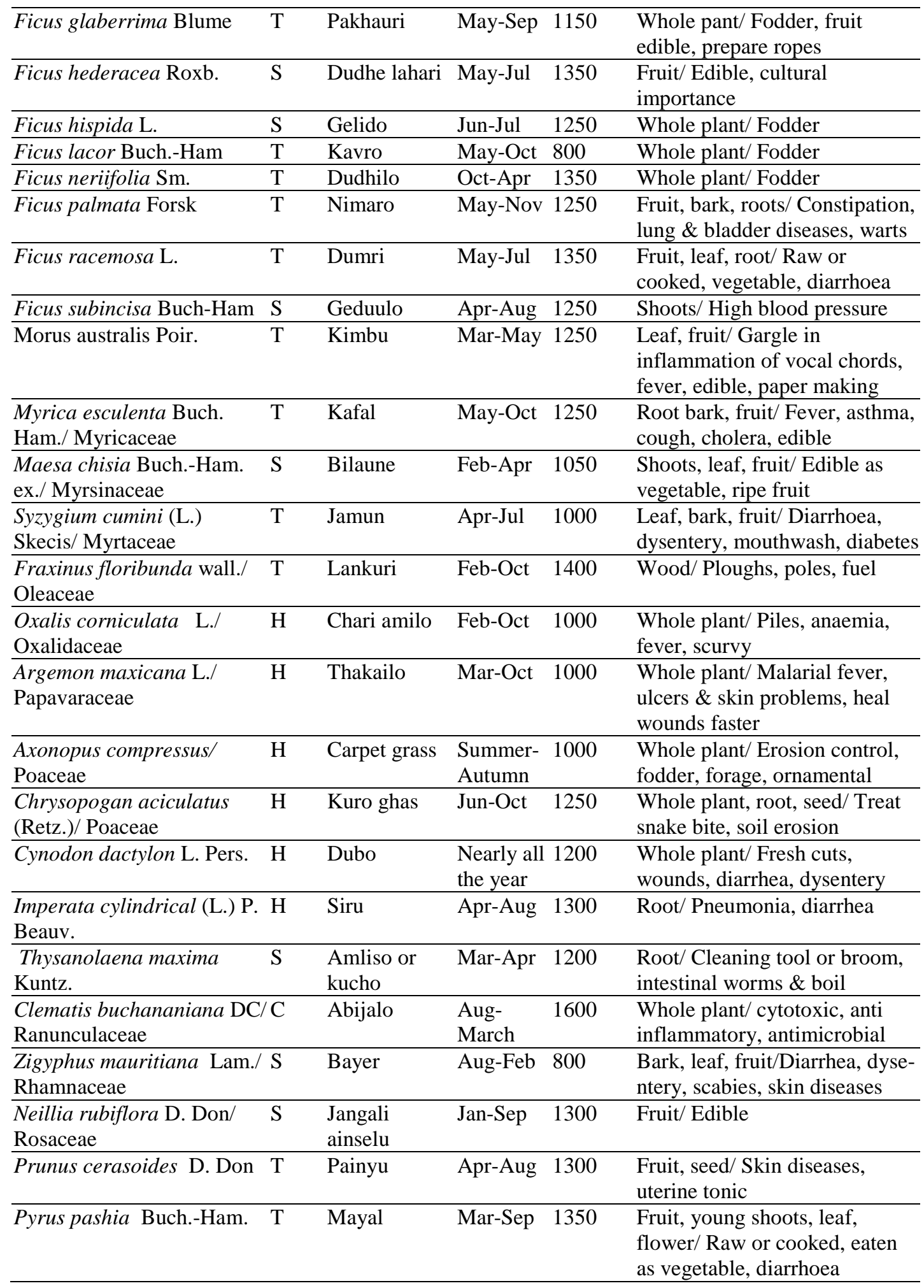




\begin{tabular}{|c|c|c|c|c|}
\hline Rubus ellipticus Sm. & Ainselu & Feb-Jul & 1100 & $\begin{array}{l}\text { Root, bark/ diarrhea, dysentery, } \\
\text { wounds, gastric }\end{array}$ \\
\hline $\begin{array}{l}\text { Rubia cordifolia } \text { Roxb./ } \\
\text { Rubiaceae }\end{array}$ & Majhito & Jul-Oct & 1100 & $\begin{array}{l}\text { Root, whole plant/ Natural dye, } \\
\text { fruit edible }\end{array}$ \\
\hline $\begin{array}{l}\text { Zanthoxylum armatum DC./S } \\
\text { Rutaceae }\end{array}$ & Timur & - & 1300 & $\begin{array}{l}\text { Whole plant/ Fever, cholera, } \\
\text { stomach pain, poison, spices }\end{array}$ \\
\hline $\begin{array}{l}\text { Osyris wightiana Wall.ex } \\
\text { wight/ Santalaceae }\end{array}$ & Nundhiki & Jan-Dec & 1500 & $\begin{array}{l}\text { Root, bark, fruit/ Atonics in } \\
\text { soup, fruit, treating diarrhorea }\end{array}$ \\
\hline $\begin{array}{l}\text { Diploknema butyracea } \\
\text { (Roxb.) H.J. Lam/ } \\
\text { Sapotaceae }\end{array}$ & Chiuri & July-Aug & 1100 & $\begin{array}{l}\text { Fruit, seed, leaf, bark/ Soap, } \\
\text { oil, candle, ghee, making } \\
\text { Tapari }\end{array}$ \\
\hline $\begin{array}{l}\text { Solanum aculeatissimum } \\
\text { Jacq./ Solanaceae }\end{array}$ & Kantakari & Aug-May & 800 & $\begin{array}{l}\text { Seed, root/ Ulcerated nose, } \\
\text { worms }\end{array}$ \\
\hline Solanum surattense Burm. $\mathrm{H}$ & Kantakari & Jan-Apr & 800 & $\begin{array}{l}\text { Fruit, seed, root/Cough, } \\
\text { asthama, anthelmintic }\end{array}$ \\
\hline Solanum nigrum L. & Kali bihi & Dec-Mar & 1250 & $\begin{array}{l}\text { Leaf, fruit/ Dropsy, heart } \\
\text { diseases, food }\end{array}$ \\
\hline Eurya acuminate/Theaceae $\mathrm{S}$ & Jhyanu & May-june & 1600 & $\begin{array}{l}\text { Flower, leaf, branch / Edible } \\
\text { fuel, wood }\end{array}$ \\
\hline Schima wallichii (DC.) & Chilaune & $\begin{array}{l}\text { Whole } \\
\text { year }\end{array}$ & 1300 & $\begin{array}{l}\text { Bark, aerial parts/ Fish Poison, } \\
\text { uterine disorders, antifungal }\end{array}$ \\
\hline $\begin{array}{l}\text { Boehmeria platyphyla } \\
\text { Buch-Ham. ex Don var. } \\
\text { angolensis/ Urticaceae }\end{array}$ & & Jul-Oct & 1050 & Leaf/ fever \\
\hline Boehmeria regulosa Wedd $\mathrm{T}$ & Githedaar & Jul-Sep & 1500 & $\begin{array}{l}\text { Bark/ Bark juice used in cuts, } \\
\text { wounds \& body pain, bark } \\
\text { paste applied on bone fracture }\end{array}$ \\
\hline $\begin{array}{l}\text { Girardiana diversifolia } \\
\text { (Link.) Fris }\end{array}$ & $\begin{array}{l}\text { Chalne sisnu } \\
\text { or Allo }\end{array}$ & Sep-Oct & 1600 & $\begin{array}{l}\text { Leaf juice, whole plant, root/ } \\
\text { Fodder, fuel, wood }\end{array}$ \\
\hline Urtica dioica $\mathrm{L}$. & Sisnu & Sep-Mar & 1250 & $\begin{array}{l}\text { Leaf, stem, root/ Kidney stone, } \\
\text { asthama, sinusitis, allergies }\end{array}$ \\
\hline $\begin{array}{l}\text { Clerodendrum viscosum } \\
\text { Vent./ Verbenaceae }\end{array}$ & Bhate & Jan-June & 750 & $\begin{array}{l}\text { Root, flower/ Fever, kill worms } \\
\text { in stomach }\end{array}$ \\
\hline $\begin{array}{l}\text { Holmskioldia sanguinea } \\
\text { Retz. }\end{array}$ & Jhule Phool & May-Oct & 750 & $\begin{array}{l}\text { Leaf, shoot, bark/ Rheumatism, } \\
\text { dysentery, headaches }\end{array}$ \\
\hline Lantana camara $\mathrm{L}$. & Banmara & $\begin{array}{l}\text { Whole } \\
\text { year }\end{array}$ & 1300 & $\begin{array}{l}\text { Leaf, stalk/ Asthama, skin } \\
\text { itches, repels insect, leprosy }\end{array}$ \\
\hline Premna longifolia Roxb. & Gidari & May-Jul & 900 & $\begin{array}{l}\text { Root/ Anti-pyretic, liver } \\
\text { complaints, cold, rhematic }\end{array}$ \\
\hline Vitex negundo $\mathrm{L}$ & Simali & Apr-Dec & 1300 & $\begin{array}{l}\text { Leaf, root/Rheumatic swellings } \\
\text { of joints, dyspepsia, boils }\end{array}$ \\
\hline $\begin{array}{l}\text { Vitis lanata auction/ } \\
\text { Vitaceae }\end{array}$ & Ban angur & & 750 & $\begin{array}{l}\text { Fruit, leaf, flower/ Raw or dried } \\
\text { for winter use, yellow dye from } \\
\text { the fresh or dried leaves }\end{array}$ \\
\hline $\begin{array}{l}\text { Cayratia trifolia }(\mathrm{L} .) \\
\text { Domin. }\end{array}$ & Karaunja & June-Dec & 900 & $\begin{array}{l}\text { Whole plant/ Tumors, antivira, } \\
\text { anticancer \& diuretic activity }\end{array}$ \\
\hline $\begin{array}{l}\text { Costus speciosus (J. konig) S } \\
\text { Sm./ Zingiberaceae }\end{array}$ & Betlauri & Jul-Sep & 750 & $\begin{array}{l}\text { Wild edible plant, diabetes, } \\
\text { medicinal uses }\end{array}$ \\
\hline
\end{tabular}


Out of 127 collected species, more than 30 species were of medicinal importance. Some of them are Xanthoxylum armatum, Litsea cubeba, Terminalia chebula, Terminalia bellirica, Rhus javanica, Phyllanthus emblica, Vitex negundo, Justicia Adhatoda, Dichroa febrifuga,Tinospora cordifolia etc. Plants with edible fruit were Myrica esculenta, Rubus elipticus, Barberis aristata, Phyllanthus emblica, Morus australis, Ficus subincisa, F. semicordata, Brassia butyraceae etc. Common fodder plants were Ficus semicordata, F. subincisa, F. hispida, F. nerifolia, F. racemosa, F. palmata, F. semicordata, F. hispida, Morus australis, Litsea monopetala, etc. Some economically important species were Terminalia chebula, T. bellerica, Shorea robutsa, Schima wallichii, Xanthoxyllum armatum, Phyllanthus emblica, Thysanolaena maxima, Dioscorea bulbifera etc. Sapium insigne, Ageratina adenophora, Adhatoda vassica were used as green manure. Lantana

camara, Eupatorium adenophorum, Ageratum conizoids were invasive alien species. Dendrophthoe falcate was a parasite on plant.

\section{References}

Chaudhary, R.P. 1998. Biodiversity in Nepal: Status and conservation. S. Devi, Saharapur, U. P., India and Tecpress books 487/42 Soiwattanasilp Pratunam, Bangkok 10400, Thailand.

Haines, H.H. 1961 (Revd. ed.). The botany of Bihar and Orissa, vol. 3. Bishen Singh and Mahendra Pal Singh, DehraDun, India.

Hara, H. \& L.H. Williams 1979. An enumeration of the flowering plants of Nepal, vol. 2. British Museum (Nat. Hist.), London.

Hara, H., A.O. Chater \& L.H. Williams 1982. An enumeration of the flowering plants of Nepal, vol. 3. British Museum (Nat. Hist.), London.

Hara, H., W.T. Steam \& L.H. Williams 1978. An enumeration of the flowering plants of Nepal, vol. 1. British Museum (Nat. Hist.), London.

Hooker, J.D. 1854. Himalayan Journal, vols. 1-2. Today and Tomorrow printers.

Hooker, J.D. 1883-1897. Flora of British India, vols. 1-7. London.

Lawrence, G.H.M. 1965. Taxonomy of vascular plants. Macmillian Company, New York.

Press, J.R., K.K. Shrestha \& D.A. Sutton 2000. Annotated checklist of the flowering plants of Nepal. The Natural History Museum, London.

Sapkota, P.P. 2000. Ecological study and traditional uses of medicinal plants at Malika forest, Baglung. M.Sc. Thesis, Central Department of Botany, T.U., Kirtipur, Kathmandu.

Shrestha, T.B. \& R.M. Joshi 1996. Rare, endemic and endangered plants of Nepal. WWF Nepal Program, Kathmandu.

Siwakoti, M. \& S.K. Varma 1996. Medicinal plants of the terai of eastern Nepal. J. Econ. Tax. bot. Addi. Ser. 12: 423-438.

Siwakoti, M. \& S.K. Varma 1999. Plant diversity of eastern Nepal: flora of plains of eastern Nepal. Bishen Singh and Mahendra Pal Singh, Dehra Dun, India. 491p.

Stainton, J.D. 1972. Forest of Nepal. John Murray, London 